\title{
GERAÇÃO DE FINOS NO BRANQUEAMENTO DE PASTA KRAFT DE EUCALIPTO E SEU EFEITO NAS PROPRIEDADES DO PAPEL ${ }^{1}$
}

Jaqueline Silveira Comelato², Gustavo Ventorim³ ${ }^{3}$ José Cláudio Caraschi $^{3}$ e Ivan Rodrigues dos Santos ${ }^{2}$

\begin{abstract}
RESUMO - Este trabalho teve por objetivo avaliar a quantidade de finos gerada por diferentes reagentes de branqueamento e seu efeito nas propriedades físicas e mecânicas da celulose kraft de eucalipto. A polpa foi branqueada por quatro sequências diferentes. Parte das amostras foi classificada em equipamento BauerMcNett, sendo a parte não classificada (global) refinada para $40^{\circ} \mathrm{SR}$, em moinho laboratorial PFI. As duas maiores porções de cada amostra proveniente da classificação foram também refinadas com o mesmo número de revoluções que a sua amostra global. Realizaram-se ensaios físicos e mecânicos das amostras refinadas, e suas fibras foram analisadas em equipamento FQA (Fiber Quality Analyser), antes e depois do refino. A maior quantidade de finos foi observada nas polpas refinadas e na sequência-referência. Os resultados de tração foram mais elevados e significativos nas amostras globais; as polpas classificadas não apresentaram diferença entre si, sendo atribuída a maior resistência à tração na presença de finos. A resistência ao rasgo foi afetada pelo comprimento das fibras e não pelo teor de finos. Os maiores valores de ascensão capilar Klemm ocorreram nas amostras classificadas em razão da ausência de finos e do maior comprimento de fibras.
\end{abstract}

Palavras-chave: Classificação de fibras, Refino, Propriedades físicas e mecânicas.

\section{GENARATIONS OF FINES IN EUCALYPTUS KRAFT PULP BLEACHING AND THEIR EFFECT ON PAPER PROPERTIES}

\begin{abstract}
The objective of this work was to evaluate the generation of fines by different bleaching reagents and its effect on physical and mechanical properties of eucalyptus kraft pulp. The pulp was bleached by four different sequences. Some of these samples was classified in Bauer-McNett equipment. The unclassified part of the pulp (global) was refined to $40{ }^{\circ} S R$ in a laboratory PFI mill. The two major classified portions of each sample were also refined at the same revolutions as its global sample. Physical and mechanical tests were performed for both classified and unclassified samples, and their fibers were analyzed in FQA (Fiber Quality Analyzer), before and after refining. The greater amount of fines was observed for the refined pulp and the reference sequence. The tensile results were more significant for the global sample, classified pulps showed no difference between them being given the highest tensile strength to the presence of fines. The tear resistance was affected by the length of the fibers and not by the content of fines. The highest values of capillarity for the Klemm samples were classified due to the absence of fines and fibers of greater length.
\end{abstract}

Keywords: Fiber classification, Physical and mechanical properties, Refining.

\footnotetext{
${ }^{1}$ Recebido em 21.05.2012 aceito para publicação em 20.12.2012.

${ }^{2}$ Progrma de Pós-Graduação em Engenharia Mecância na Universidade Estadual Paulista, UNESP, Faculdade de Engenharia de Guaratinguetá.E-mail:<jaquecomelato@hotmail.com>e <ivansantos.unesp@gmail.com>.

${ }^{3}$ Universidade Estadual Paulista Júlio de Mesquita Filho, Campus Experimental de Itapeva, SP, Brasil. E-mail: <ventorim@itapeva.unesp.br>e < caraschi@itapeva.unesp.br>.
}

Revista Árvore, Viçosa-MG, v.37, n.1, p.181-189, 2013 


\section{INTRODUÇÃO}

Durante o processo de polpação da madeira, as fibras são individualizadas e separadas da matriz madeira, sendo geradas frações de fibras. Essas frações são denominadas "finos" celulósicos e consideradas de vital importância durante a fabricação do papel, pois estão relacionadas com o baixo rendimento produtivo, como também causam inúmeros danos e prejuízos às indústrias de celulose de eucalipto. Os finos são formados durante todo o processo produtivo, desde o cozimento até a etapa de formação da folha. Durante o branqueamento da celulose, os reagentes químicos utilizados nas sequências de branqueamento afetam as propriedades dos polissacarídeos, diminuindo o comprimento das cadeias (KANGAS; KLEEN, 2004). O gerenciamento dos finos constitui aspecto indispensável para as indústrias de celulose, pois em grandes proporções, ou em extremamente baixas proporções, afeta as propriedades de resistência do papel.

São considerados "finos" a fração de polpa que atravessa uma malha de 200 mesh. Isso equivale a um tamanho médio de partícula de 75 ìm (TAPPI $233 \mathrm{~cm}$ 06 e TAPPI 261-cm-00). Os finos são constituídos por células de raio, partes da lamela média, células de vaso e fibrilas e contêm grande quantidade de lignina e extrativos; apenas $23 \%$ da superfície dos finos são compostos por polissacarídeos, ricos em grupos carboxílicos, por causa das pectinas e também dos ácidos metilglicurônicos das xilanas (KLEEN et al., 2003). Polpa com baixo teor de finos apresenta menores teores de extrativos e metais, maior alvura final no branqueamento e maior estabilidade de alvura (ASIKAINEN et al., 2010). Em polpas de coníferas, a remoção dos finos pode aumentar em até $2 \%$ ISO a alvura de polpas em branqueamento e ocasionar a economia de peróxido de hidrogênio de até $7 \mathrm{~kg} / \mathrm{t}$ de polpa (BÄCKSTRÖM; BRÄNNVALL, 1999).

A etapa mecânica de refino exerce grande influência na estrutura física da fibra e na geração de finos. O refino tem por objetivo melhorar as interações entre as fibras, porém resulta em alterações estruturais, incluindo o encurtamento da fibra, fibrilação interna e externa, como também a produção de finos na polpa de celulose (KROGERUS; FAGERHOLM, 2002). A refinação da celulose geralmente eleva a resistência física e reduz as propriedades ópticas da folha, devido à maior conformação e uniformidade das fibras (BANAVATH et al., 2011; KEREKES, 2005; LUNDIN

Revista Árvore, Viçosa-MG, v.37, n.1, p.181-189, 2013 et al., 2009), tanto para fibras virgens quanto para fibras recicladas (CHEN et al., 2012). Entretanto, ao atingirem grau máximo, as propriedades tendem a se estabilizar e, se o refino for prolongado, pode levar ao declínio das propriedades (GIGAC; FIŠEROVÁ, 2008; LUNDIN et al., 2009).

Os finos celulósicos melhoram praticamente todas as propriedades de resistência das folhas de papel de eucalipto, pois a área de contato entre as fibras é melhorada. Também podem ter algum efeito positivo na opacidade, mas isso vai depender do nível de refino e da densidade e compactação da folha (KROGERUS; FAGERHOLM, 2002; TAIPALE et al., 2010). Estudos indicaram que, a partir da seleção adequada da matériaprima e das condições de processo, obtém-se um aprimoramento das propriedades de resistência sem que, simultaneamente, diminua a drenagem da polpa (TAIPALE et al., 2010).

Muitos pesquisadores estudaram a classificação de fibras e o teor de finos em Bauer-McNett (GOODING; OLSON, 2001; SILVA et al., 1997; CARVALHO et al., 1997). Os resultados podem ser variados, já que as operações de polpação e branqueamento modificam a natureza dos constituintes químicos das fibras, vasos e finos celulósicos.

A etapa de branqueamento tende a diminuir o potencial de resistência da fibra, gerando também finos. Cada reagente utilizado nas sequências de branqueamento de polpa kraft afeta diferentemente as propriedades da celulose produzida. Isso ocorre em razão, principalmente, do uso de reagentes como oxigênio e seus derivados (ozônio, peróxido de hidrogênio e perácidos), os quais são menos seletivos para a preservação do potencial de resistência da fibra (DENCE; REEVE, 1996). Resta saber se diferentes sequências de branqueamento possuem influência sobre a qualidade dos finos produzidos e se estes afetam as propriedades físicas e mecânicas do papel. Desse modo, este trabalho foi realizado para avaliar a geração de finos proporcionada por diferentes sequências de branqueamento e verificar o efeito dos finos nas propriedades físicas e mecânicas da polpa de celulose kraft de eucalipto, classificada e não classificada.

\section{MATERIAL E MÉTODOS}

Uma amostra de polpa industrial de eucalipto, prédeslignificada com oxigênio, foi utilizada neste estudo. As características iniciais da polpa foram: número kappa

\footnotetext{
Revista Árvore, Viçosa-MG, v.37, n.1, p.181-189, 2013
} 
9,8 , alvura $60,2 \%$ ISO e viscosidade $28,3 \mathrm{cP}$. Foram realizadas quatro sequências de branqueamento: $\mathrm{D}(\mathrm{E}+\mathrm{P}) \mathrm{DD}$ (referência), $\mathrm{A}_{\mathrm{HT}} \mathrm{D}(\mathrm{E}+\mathrm{P}) \mathrm{DD}, \mathrm{D}(\mathrm{E}+\mathrm{P}) \mathrm{D}(\mathrm{PO})$ e $P_{\mathrm{X}}(\mathrm{E}+\mathrm{P}) \mathrm{DD}$, cujas condições estão apresentadas na Tabela 1, com a ressalva de que essas sequências se divergiram apenas por um dos seguintes estágios: dióxido de cloro (D), ácido sulfúrico $\left(\mathrm{A}_{\mathrm{HT}}\right)$, peróxido de hidrogênio pressurizado $(\mathrm{PO})$ e ácido de Caro $\left(\mathrm{P}_{\mathrm{X}}\right)$. Todos os estágios foram simulados em laboratório.

O branqueamento com os estágios não pressurizados foram efetuados em sacos de polietileno com amostras representativas de polpa absolutamente secas. $\mathrm{O}$ licor de branqueamento foi adicionado à polpa em temperatura ambiente. $\mathrm{O}$ requerimento de $\mathrm{NaOH}$ ou $\mathrm{H}_{2} \mathrm{SO}_{4}$ para controle de $\mathrm{pH}$ foi realizado em estudo prévio com miniamostras de polpa. Terminada a reação, foram extraídas amostras do licor residual para análises de $\mathrm{pH}$ e residual (quando aplicável). A polpa foi lavada com o equivalente a $9 \mathrm{~mL}$ de água por grama de polpa absolutamente seca.

O estágio com peróxido pressurizado foi efetuado em um digestor com quatro reatores individuais de aço inox, com capacidade para 1,5 L cada, com ajuste de temperatura e pressão. Em cada cápsula foram colocados $110,0 \mathrm{~g}$ a.s. de polpa. Foi adicionada à polpa uma mistura de água para ajuste da consistência, ou seja, $\mathrm{NaOH}$ para ajuste do $\mathrm{pH}$ e $\mathrm{H}_{2} \mathrm{O}_{2}$ para o branqueamento. Após atingir a temperatura estabelecida, sob o efeito da rotação constante do digestor, foi aplicada a carga de oxigênio, mantendo a pressão de $5 \mathrm{~atm}$. Ao término da deslignificação, a pressão do digestor foi aliviada para retirar a polpa das cápsulas. Foram extraídas amostras do licor residual para análise de pH. Em seguida, a polpa foi lavada com o equivalente a $9 \mathrm{~mL}$ de água por grama de polpa absolutamente seca.

Com o objetivo de quantificar a dimensão das fibras das amostras, foi utilizado um classificador de fibras BauerMcNett, que é capaz de separar amostras de fibras em frações, de acordo com a norma TAPPI T $233 \mathrm{~cm}-95$. Foram realizadas, em média, 25 repetições de classificação de cada uma das quatro amostras branqueadas.

As polpas não classificadas foram refinadas a partir de curvas de refino em moinho PFI. Foi estabelecido que os testes físicos e mecânicos fossem realizados apenas para as polpas com $40^{\circ}$ Schopper-Riegler ( $\left.{ }^{\circ} \mathrm{SR}\right)$. As polpas classificadas também foram refinadas, porém apenas as duas maiores porções de polpas retidas nas malhas do classificador, que foram refinadas ao mesmo número de revoluções que a sua amostra não classificada. Após o refino e a determinação do ${ }^{\circ} \mathrm{SR}$, foram confeccionadas 20 folhas de cada amostra e realizados os ensaios físicos e mecânicos. As análises da polpa produzida e as propriedades físicas e mecânicas foram efetuadas seguindo-se os procedimentos analíticos da TAPPI e ISO. Os ensaios físicos e mecânicos foram realizados com 10 repetições cada. Os resultados foram analisados através da análise de variância (ANOVA) e do teste de comparação de médias pelo teste de Tukey a 5\% de significância (95\% de probabilidade).

Tabela 1 - Condições gerais do processo de branqueamento. Table 1 -Bleaching general conditions.

\begin{tabular}{|c|c|c|c|c|c|c|c|}
\hline \multirow[t]{2}{*}{ Condições } & \multicolumn{7}{|c|}{ Estágios de branqueamento } \\
\hline & $\mathrm{D}_{0}$ & $\mathrm{~A}_{\mathrm{HT}}$ & $P_{x}$ & $(\mathrm{E}+\mathrm{P})$ & $\mathrm{D}_{1}$ & $\mathrm{D}_{2}$ & $\mathrm{PO}$ \\
\hline Consistência (\%) & 10 & 10 & 10 & 10 & 10 & 10 & 10 \\
\hline Tempo (min.) & 30 & 120 & 30 & 60 & 180 & 180 & 60 \\
\hline Temperatura $\left({ }^{\circ} \mathrm{C}\right)$ & 60 & 95 & 60 & 70 & 70 & 70 & 105 \\
\hline Pressão $(\mathrm{kPa})$ & - & - & - & - & - & - & 500 \\
\hline $\mathrm{pH}$ final & 2,7 & 2,0 & 3,0 & 11,2 & 3,8 & 4,1 & 10,5 \\
\hline $\mathrm{NaOH}, \mathrm{kg} / \mathrm{t}$ & - & - & $* *$ & 8,0 & - & $* *$ & 8,0 \\
\hline $\mathrm{H}_{2} \mathrm{SO}_{4}, \mathrm{~kg} / \mathrm{t}$ & $* *$ & $* *$ & & - & $* *$ & - & - \\
\hline $\mathrm{O}_{2}, \%$ & - & - & - & - & - & - & 2,0 \\
\hline $\mathrm{ClO}_{2}, \mathrm{~kg} / \mathrm{t}$ como $\mathrm{Cl}_{2}$ & $\mathrm{FK}^{1}=0,2$ & - & - & - & $*$ & $*$ & - \\
\hline $\mathrm{H}_{2} \mathrm{O}_{2}, \mathrm{~kg} / \mathrm{t}$ & - & - & - & 3,0 & - & - & 3,0 \\
\hline $\mathrm{P}_{\mathrm{X}}, \mathrm{kg} / \mathrm{t}$ & - & - & 5,0 & - & - & - & - \\
\hline
\end{tabular}

${ }^{1}$ Fator kappa. $*$ Dosagem otimizada para obter alvura de $90 \pm 0,5 \%$ ISO. $* *$ Dosagem otimizada para ajuste de pH. 
As fibras da polpa branqueada foram avaliadas utilizando-se o equipamento FQA - Fiber Quality Analyser, modelo LDA02, do fabricante OpTest. Os seguintes parâmetros foram analisados: comprimento ponderado em comprimento da fibra, a coarseness da fibra e teor de finos. Essas determinações foram efetuadas em 16 amostras de polpas branqueadas, sendo quatro globais e quatro classificadas e retidas na malha de 50 mesh do classificador, com duas repetições cada.

\section{RESULTADOS}

\subsection{Branqueamento}

Os resultados experimentais, obtidos em cada estágio de branqueamento, das alternativas $\mathrm{D}(\mathrm{E}+\mathrm{P}) \mathrm{DD}$ (referência), $\mathrm{A}_{\mathrm{HT}} \mathrm{D}(\mathrm{E}+\mathrm{P}) \mathrm{DD}, \mathrm{D}(\mathrm{E}+\mathrm{P}) \mathrm{D}(\mathrm{PO}) \mathrm{eP}_{\mathrm{X}}(\mathrm{E}+\mathrm{P}) \mathrm{DD}$ são apresentados na Tabela 2. Os resultados dos branqueamentos das quatro amostras foram interpretados com base nos parâmetros de qualidade da polpa branqueada, como valores de viscosidade e reversão de alvura.

Todas as polpas foram branqueadas para que se atingissem $90 \pm 0,5 \%$ ISO de alvura. Na Tabela 2, verifica-se que a reversão de alvura foi menor na polpa com o estágio $\mathrm{A}_{\mathrm{HT}}$, apresentando a reversão de apenas $1,1 \%$ ISO. A sequência $\mathrm{A}_{\mathrm{HT}} \mathrm{D}(\mathrm{E}+\mathrm{P}) \mathrm{DD}$ produziu polpa com viscosidade de $21,8 \mathrm{cP}, 10,0 \%$ mais baixa que a referência, de 24,2 cP. Os resultados indicaram que os estágios de hidrólise ácida, peróxido pressurizado e com perácido reduziram o tamanho médio das cadeias de carboidratos em relação à sequência referência.

\subsection{Classificação e análise de fibras}

Na Figura 1, verifica-se que a maior quantidade de polpa foi retida na peneira com malha de 50 mesh para todas as sequências. Na sequência referência, a segunda maior quantidade de polpa ficou retida na malha de 30 mesh, como ocorreu também para a amostra com estágio $\mathrm{P}_{\mathrm{X}}$. Já as polpas branqueadas por $\mathrm{PO} \mathrm{e}$ $\mathrm{A}_{\mathrm{HT}}$ tiveram as maiores quantidades de polpa retidas nas malhas de 50 e 100 mesh.

A maior porção de finos foi para a sequência com o estágio $\mathrm{A}_{\mathrm{HT}}$, equivalente a $7,17 \%$, mostrando que esse estágio foi o que causou maior hidrólise dos carboidratos.

O teor de finos, além de ser quantificado pelo Bauer-McNett, foi mensurado também pelo analisador de fibras FQA. Foram denominadas R Global e R50; A Global e A50; P Global e P50; X Global; e X50 as amostras não classificadas (global) e as amostras retidas nas malhas de 50 mesh do classificador, para as sequências $\mathrm{D}(\mathrm{E}+\mathrm{P}) \mathrm{DD}, \mathrm{A}_{\mathrm{HT}} \mathrm{D}(\mathrm{E}+\mathrm{P}) \mathrm{DD}, \mathrm{D}(\mathrm{E}+\mathrm{P}) \mathrm{D}(\mathrm{PO})$ e $P_{X}(E+P) D D$, respectivamente. Verifica-se, na Figura 2, que o teor de finos foi significativamente maior nas polpas refinadas.

Tabela 2 - Características das polpas branqueadas.

Table 2 - Characteristics of bleached pulp.

\begin{tabular}{|c|c|c|c|c|c|c|c|c|}
\hline \multirow{2}{*}{ Resultados } & \multicolumn{2}{|c|}{$\mathrm{D}(\mathrm{E}+\mathrm{P}) \mathrm{DD}$} & \multicolumn{2}{|c|}{$\mathrm{A}_{\mathrm{HT}} \mathrm{D}(\mathrm{E}+\mathrm{P}) \mathrm{DD}$} & \multicolumn{2}{|c|}{$\mathrm{D}(\mathrm{E}+\mathrm{P}) \mathrm{D}(\mathrm{PO})$} & \multicolumn{2}{|c|}{$\mathrm{PX}(\mathrm{E}+\mathrm{P}) \mathrm{DD}$} \\
\hline & Global & $\mathrm{R} 50$ & Global & A50 & Global & P50 & Global & X50 \\
\hline Alvura A.D., \% ISO & 90,2 & - & 90,4 & - & 90,9 & - & 89,5 & - \\
\hline Rev. de Alvura, \% ISO & 2,9 & - & 1,1 & - & 1,3 & - & 1,8 & - \\
\hline Viscosidade, $\mathrm{cP}$ & 24,2 & - & 21,8 & - & 22,2 & - & 22,6 & - \\
\hline $\mathrm{ClO}_{2}$ como $\mathrm{Cl}_{2}, \mathrm{~kg} / \mathrm{t}$ & 35,6 & - & 30,6 & - & 32,6 & - & 29 & - \\
\hline $\mathrm{H}_{2} \mathrm{O}_{2}, \mathrm{~kg} / \mathrm{t}$ & 3 & - & 3 & - & 6 & - & 3 & - \\
\hline $\mathrm{NaOH}, \mathrm{kg} / \mathrm{t}$ & 7,8 & - & 8 & - & 16,8 & - & 33 & - \\
\hline $\mathrm{H}_{2} \mathrm{SO}_{4}, \mathrm{~kg} / \mathrm{t}$ & 1 & - & 5 & - & 1 & - & 0 & - \\
\hline $\mathrm{P}_{\mathrm{X}}, \mathrm{kg} / \mathrm{t}$ & 0 & - & 0 & - & 0 & - & 5 & - \\
\hline $\mathrm{O}_{2}, \mathrm{~kg} / \mathrm{t}$ & 0 & - & 0 & - & 20 & - & 0 & - \\
\hline Revoluções PFI & 2300 & 2300 & 2100 & 2100 & 1500 & 1500 & 1800 & 1800 \\
\hline PFI (Wh) & 60,3 & 60,3 & 50,6 & 50,6 & 18 & 18 & 45,5 & 45,5 \\
\hline${ }^{\circ} \mathrm{SR}$ & 40 & 27 & 40 & 24 & 40 & 19 & 40 & 21 \\
\hline Comprimento, $\mathrm{mm}$ & 0,85 & 0,87 & 0,86 & 0,87 & 0,83 & 0,87 & 0,83 & 0,87 \\
\hline Finos, $\%$ & 18 & 3,1 & 11,4 & 3,9 & 10,8 & 3,1 & 13,3 & 3,4 \\
\hline Coarseness, g/100 m & 7,9 & 6,3 & 7,1 & 7,9 & 7,5 & 8,1 & 7,4 & 6,0 \\
\hline
\end{tabular}

Revista Árvore, Viçosa-MG, v.37, n.1, p.181-189, 2013 
Os diferentes processos de branqueamento produziram polpas branqueadas com teores de finos variando de 7,5 a 9,0\%. Após o refino, esse valor foi elevado para a faixa de 10,8 a $18,0 \%$. O efeito do branqueamento na produção de finos na polpa foi bastante significativo, dependendo do estágio de branqueamento utilizado. É importante mencionar que foi medido o teor de finos gerados na polpação e branqueamento e que ficaram retidos na polpa branqueada e não os finos produzidos durante o branqueamento e que saíram na água de lavagem da polpa.

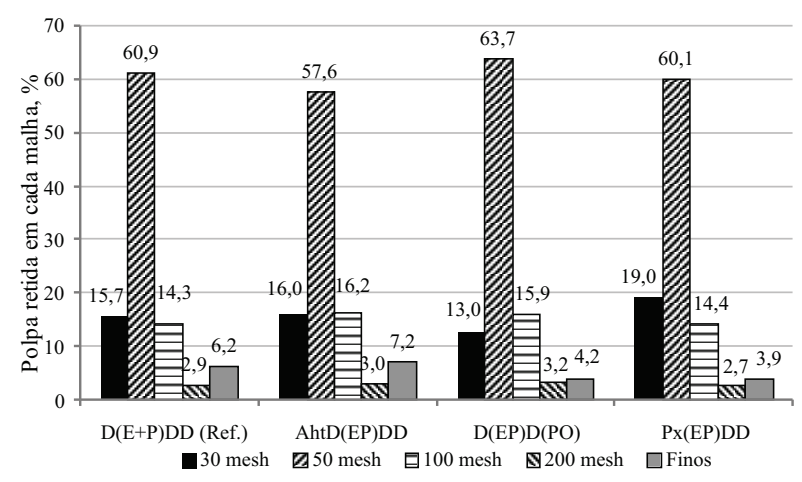

Figura 1 - Porcentagem média de massa de polpa retida em cada tanque do classificador.

Figure 1 -Average percentage of weighted pulp retained on each classifier's chamber.

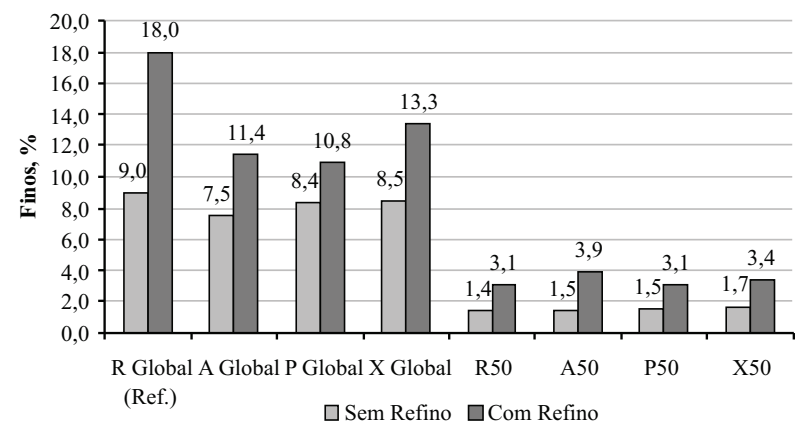

Figura 2 - Porcentagem média de finos, medida em FQA, das amostras globais e classificadas antes e depois do refino.

Figure 2-Average percentage of the fines, measured in $F Q A$, for global and classified samples, before and after refining.

\subsection{Testes físicos e mecânicos}

Os ensaios físicos e mecânicos foram analisados para as sequencias $\mathrm{D}(\mathrm{E}+\mathrm{P}) \mathrm{DD}, \mathrm{A}_{\mathrm{HT}} \mathrm{D}(\mathrm{E}+\mathrm{P}) \mathrm{DD}$, $\mathrm{D}(\mathrm{E}+\mathrm{P}) \mathrm{D}(\mathrm{PO}), \mathrm{P}_{\mathrm{X}}(\mathrm{E}+\mathrm{P}) \mathrm{DD}$ e também para a polpa classificada retida na malha de 50 mesh. Todos os ensaios físicos e mecânicos foram realizados apenas nas amostras refinadas.

Entre as amostras globais, de acordo com a Figura 3a, o índice de tração foi maior nas amostras R Global e A Global e X Global. Apesar de possuir também 40 ${ }^{\circ} \mathrm{SR}$, a polpa P Global apresentou menor resistência à tração que as demais amostras. Entre as amostras classificadas, a amostra X50 foi a que apresentou o menor índice de tração.

Apesar de classificada, a polpa R50 apresentou índice de tração estatisticamente igual à polpa R Global; o mesmo ocorreu nas polpas A Global e A50, sendo estatisticamente iguais, apesar de classificadas, com ${ }^{\circ} \mathrm{SR}$, coarseness e teores de finos menores, apresentaram a mesma resistência à tração que as polpas globais. No entanto, nas polpas $\mathrm{PO}$ e $\mathrm{P}_{\mathrm{X}}$ esse mesmo comportamento não foi observado, e P Global apresentou índice de tração 20,6\% maior que a amostra P50, enquanto a amostra X Global apresentou índice de tração 48,6\% maior que a amostra X50.

Na Figura 3b, verifica-se que o índice de rasgo das amostras globais foram estatisticamente iguais. O menor valor de índice de rasgo foi da amostra X50. Apesar de X Global apresentar o mesmo comprimento de fibra que R Global, foi observado após a classificação das fibras redução de $20,4 \%$ da polpa X50 em relação à polpa R50. O teor de finos presentes nas amostras globais, apesar de ser inferior nas polpas P Global e X Global, não interferiu no índice de rasgo do papel. Entre as amostras de 50 mesh, o maior índice de rasgo foi da R50. A amostra A50 foi considerada estatisticamente igual à R50. Apesar de as polpas classificadas apresentarem o mesmo comprimento, os estágios PO e $\mathrm{P}_{\mathrm{X}}$ afetaram negativamente a propriedade de índice de rasgo das amostras P50 e X50 em relação à polpa referência de mesmo comprimento de fibras (R50).

De acordo com a Figura 3c, foi observado maior valor de capilaridade Klemm nas amostras classificadas. Entre as globais, P Global foi a amostra que apresentou a maior ascensão capilar Klemm, enquanto as demais classificadas, as menores ascensões Klemm e

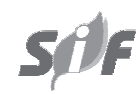

Revista Árvore, Viçosa-MG, v.37, n.1, p.181-189, 2013 

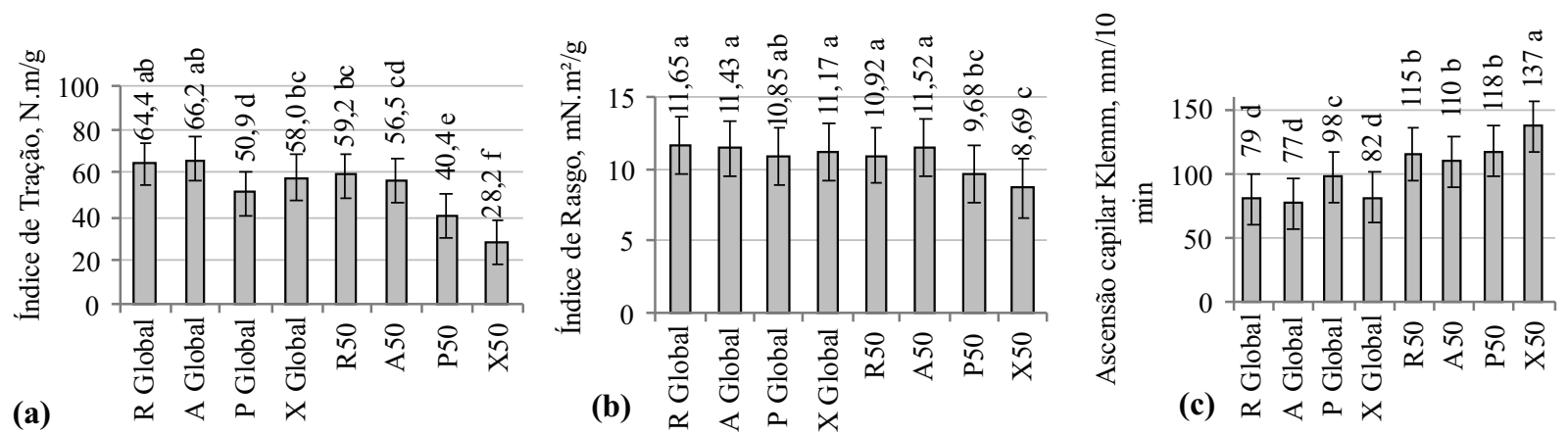

Figura 3 - Análise estatística de: a) índice de tração, b) índice de rasgo e c) ascensão capilar Klemm das amostras globais e classificadas. Médias seguidas por uma mesma letra em um mesmo conjunto de barras não apresentam diferença significativa pelo teste de Tukey a $5 \%$ de probabilidade.

Figure 3 - Statistical analysis of: a) tensile index b) tear index c) Klemm capillary rise, for global and classified sample. Means followed by the same letter do not significantly differ by the Tukey's test at $5 \%$ probability level.

estatisticamente iguais entre si. O estágio com PO foi o menos afetado para a propriedade de capilaridade entre as amostras não classificadas. Porém, nesta análise, os maiores valores de capilaridade são das polpas classificadas. A polpa $\mathrm{P}_{\mathrm{X}}$ apresentou o maior valor entre as demais amostras, equivalente a $137 \mathrm{~mm} / 10 \mathrm{~min}$. A polpa branqueada com perácido foi menos afetada em relação à propriedade de ascensão capilar.

\section{DISCUSSÃO}

\subsection{Branqueamento}

Entre as sequências analisadas, verificou-se que a reversão de alvura foi menor na amostra com o estágio $\mathrm{A}_{\mathrm{HT}}$, já que o estágio ácido remove os ácidos hexenurônicos, proporcionando maior estabilidade de alvura ( $1,1 \%$ ISO), o que pode ser explicado pelo fato de a polpa do estágio $\mathrm{A}_{\mathrm{HT}}$, realizado em altas temperaturas e baixo $\mathrm{pH}$, remover praticamente todos os metais de transição, principalmente o ferro, o qual é o principal causador da reversão de alvura (EIRAS et al., 2005; DUARTE; LACHENAL, 2002; GULLICHSEN; SODERHJELM, 1984). A reversão de alvura também foi baixa na sequência com estágio $\mathrm{PO}$, o que pode ser explicado pela alta estabilidade de alvura do peróxido de hidrogênio presente nesse estágio (ZERONIAN; INGLESBY, 1995; COLODETTE et al., 2004; EIRAS et al., 2005; OLIVEIRA et al., 2006), no entanto não foi mais baixa que a sequência $A_{\mathrm{HT}}$, pois o estágio com peróxido pressurizado com oxigênio leva à substancial formação de grupos carboxílicos, causadores de instabilidade de alvura (MAIA; COLODETTE, 2003).
Esse resultado também está de acordo com os observados por Karim et al. (2011), quando foi verificado que a substituição do estágio D no final da sequência por PO aumenta a estabilidade de alvura, já que o estágio de peróxido pressurizado, no final da sequência de branqueamento, possui o poder de estabilizar os grupos carbonílicos na celulose e hemicelulose, gerados durante o branqueamento.

A sequência $A_{H T} D(E+P) D D$ produziu polpa com viscosidade de $21,8 \mathrm{cP}$, significativamente menor que a referência, de 24,2 cP. Os resultados indicaram simplesmente que os estágios de hidrólise ácida, peróxido pressurizado e com perácido reduziram o tamanho médio das cadeias de polissacarídeos em relação à sequência referência. Porém, segundo D’Almeida (1986), a viscosidade não é um parâmetro confiável para a verificação da perda de resistência da polpa. Somente os testes físicos e mecânicos das fibras podem indicar se, realmente, houve verdadeira perda de resistência da fibra.

\subsection{Classificação e análise de fibras}

Principalmente, o estágio com peróxido pressurizado afetou a polpa, fazendo que aumentasse o percentual de fibras mais curtas. Uma possível explicação da diminuição do tamanho da fibra é a despolimerização terminal no polímero de celulose. De acordo com Silva et al. (1997), a classificação de fibras em Bauer-McNett sofre elevadas variações, dependendo das características químicas, físicas e anatômicas da madeira e do processo de branqueamento utilizado. 
As amostras branqueadas pelas sequências com os estágios $\mathrm{PO}$ e $\mathrm{P}_{\mathrm{X}}$ apresentaram porcentagem de finos estatisticamente iguais, porém inferiores à referência. Uma possível explicação da sequência com estágio PO ter baixo teor de finos é a solubilização destes durante o branqueamento com peróxido pressurizado. No caso do $\mathrm{P}_{\mathrm{X}}$, os finos gerados foram solubilizados no estágio $(\mathrm{E}+\mathrm{P})$.

O refino, apesar de melhorar quase todas as propriedades mecânicas do papel, causa ruptura nas fibras, aumentado, assim, seu teor de finos (MILES; KARNIS, 1995; KEREKES, 2005; LUNDIN et al., 2009). O teor de finos medido pelo classificador Bauer-McNett e pelo FQA é muito diferente, não sendo possível a determinação do mesmo valor de porcentagem de finos nos dois equipamentos. O primeiro envolve um método gravimétrico e o segundo, um método de análise de pequena fração de polpa em um equipamento de análise por imagem. Na polpa não classificada, o processo de refino foi responsável pela diminuição do comprimento médio das fibras, pois se aumentou o rompimento das células, aumentando também o teor de fragmentos menores.

\subsection{Propriedades físicas e mecânicas}

Foi verificado que o índice de tração das polpas globais foi maiores que das polpas classificadas devido ao baixo teor de finos, pois a sua presença aumenta a coesão entre as fibras, apresentando maior resistência à tração (ASIKAINEN et al., 2010; LEE et al., 2011). A polpa R50 apresentou I.T 8,1\% menor que a R Global. A resistência à tração é controlada por fatores como a resistência individual das fibras, comprimento médio das fibras e com a formação e estrutura da folha. $\mathrm{O}$ valor de resistência à tração de uma folha malformada será menor, pois a ruptura ocorre na área mais fraca (NAZHAD et al., 2000). Apesar de a polpa R50 possuir comprimento de fibra similar ao da polpa não classificada, menor resistência à tração foi observado nessa amostra. Portanto, fica clara a má interação entre as fibras, causada pela malformação na folha devido à ausência dos finos. Miles e Karnis (1995), descrevendo a relação entre as características da madeira e o consumo de energia, relataram que a redução da coarseness durante o refino aumenta a flexibilidade das fibras e a produção de finos, ambos com efeitos no grau de refino. Isso também pode ser visualizado na Tabela 2 , em que, para um mesmo ${ }^{\circ} \mathrm{SR}$, o número de revoluções das polpas varia. Esse fato permite inferir que polpas com fibras de menores dimensões, por serem mais frágeis, sofrem maior ação do refino, desprendendo mais suas camadas superficiais e gerando mais finos e, assim, alcançando aumento no grau de refino $\left({ }^{\circ} \mathrm{SR}\right)$ dessas polpas.

Apesar de as fibras classificadas apresentarem a homogeneidade entre seus tamanhos, a sequência de branqueamento com PO afetou negativamente a resistência à tração, devido à baixa seletividade desse estágio em comparação com o estágio de dióxido de cloro da polpa referência. Entre as polpas classificadas, a polpa que apresentou menor resistência à tração em relação à referência foi a polpa branqueada com o perácido. Esse resultado pode ser referente ao menor ${ }^{\circ} \mathrm{SR}$ encontrado dessas amostras e ao menor teor de finos.

$O$ índice de rasgo foi fortemente influenciado pelo comprimento das fibras. Autores como El-Sharkawy et al. (2008), Hakanen e Hartler (1995) e Page (1994) observaram relação linear entre índice de rasgo e comprimento médio da fibra. Esse comportamento de resistência ao rasgo é esperado, uma vez que o comprimento médio está relacionado com o trabalho necessário para remover fibras da estrutura da folha e com a energia elástica dissipada no rompimento da folha. Esse comportamento foi observado nesta amostra, já que as amostras que apresentaram comprimento ponderado de fibras semelhantes também mostraram valores de resistência ao rasgo estatisticamente iguais.

Apesar de a hidrólise ácida ser mais agressiva aos carboidratos, nenhuma diferença foi encontrada em relação à polpa R50 após a classificação das fibras, por apresentarem tamanhos de fibras iguais. O teor de finos não apresentou influência na propriedade de índice de rasgo das amostras. O maior índice de rasgo da polpa classificada e com maior comprimento de fibras foi também observado no estudo de Carvalho et al. (1997).

Como os finos presentes na polpa global ocupam os espaços vazios nas amostras classificadas, percebe-se o forte impacto da ausência desses na propriedade de capilaridade do papel. A propriedade física de capilaridade está relacionada com a capacidade de absorção de água pelas fibras e com a quantidade e tamanho dos espaços vazios entre elas (LEE et al., 2011). Foi verificado que as polpas classificadas apresentaram maior capilaridade em relação às polpas

Revista Árvore, Viçosa-MG, v.37, n.1, p.181-189, 2013 
não classificadas, pois possuem menor teor de finos devido à classificação. A polpa P Global apresentou capilaridade superior entre as não classificadas, pois foi a polpa com o menor número de revoluções no refino. Com este, a estrutura do papel torna-se mais consolidada, diminuindo o diâmetro médio aparente dos capilares e do lúmen das fibras no papel. Assim, o transporte ascendente de água pela estrutura fibrosa é reduzido, diminuindo a capilaridade Klemm (FERREIRA et al., 1997; SANTOS; SANSÍGOLO, 2007).

A maior ascensão capilar Klemm ocorreu na amostra X50, entre as classificadas. Quanto mais espaço entre as fibras da folha, maior a ascensão capilar. A ausência de finos nas amostras classificadas contribuiu para que apresentasse maior ascensão capilar Klemm, devido à alta porosidade apresentada pelas folhas, comportamento também observado nos estudos de Krogerus e Fagerholm (2002) e Lee et al. (2011). A alta capilaridade encontrada nas polpas classificadas, especialmente nas polpas com maiores comprimentos de fibras, pode interessar aos fabricantes de papel absorvente, pois corresponde, também, à alta maciez das folhas.

\section{CONCLUSÃO}

Os diferentes estágios de branqueamento geraram polpas com diferentes teores de finos, com comportamentos distintos durante a classificação das fibras e com diferenças entre as propriedades físicas e mecânicas.

A resistência à tração das polpas foi afetada pelo teor de finos. Maior resistência à tração foi verificada em polpas com maiores teores de finos.

A resistência ao rasgo foi afetada pelo comprimento das fibras e não pelo teor de finos. Maiores comprimentos de fibras apresentaram maiores índices de rasgo. $\mathrm{Na}$ amostra de 50 mesh, o índice de rasgo foi afetado pelos estágios $\mathrm{PO}$ e $\mathrm{P}_{\mathrm{X}}$, apresentando menores valores em relação às polpas globais e classificadas com 50 mesh.

Os maiores valores de ascensão capilar Klemm foram apresentados pelas amostras classificadas, devido à ausência de finos e ao maior comprimento das fibras. O maior valor de capilaridade foi observado na amostra $\mathrm{P}_{\mathrm{X}}$ classificada na malha de 50 mesh, em comparação com as polpas globais e classificadas na mesma malha.

\section{REFERÊNCIAS}

ASIKAINEN, S. et al. Effect of birch kraft pulp primary fines on bleaching and sheet properties. Bioresources, v.5, n.4, p.2173-2183, 2010.

BÄCKSTRÖM, M.; BRÄNNVALL, E. Effect of primary fines on cooking and TCF-bleaching. Nordic Pulp and Paper Research Journal, v.14, n.3, p.209-213, 1999.

BANAVATH, H. N.; BHARDWAJ, N. K.; RAY, A.K. A comparative study of the effect of refining on charge of various pulps. Bioresource Technology, v.102, n.6, p.4544-4551, 2011.

CARVALHO, M. G.; MARTINS, A. A.;

FIGUEREDO, M. M. Fracionamento de pasta kraft de eucalipto: caracterização físico-química e desempenho papeleiro. O Papel, v.58, n.7, p. 83-86, 1997.

CHEN, Y. et al. Effect of beating on recycled properties of unbleached eucalyptus cellulose fiber. Carbohydrate Polymers, v.87, n.1, p.730-736, 2012.

COLODETTE, J. L. et al. Influence of eucalypt wood supply on pulp brightness stability.

Appita Journal, v.57, n.6, p.481-487, 2004.

D'ALMEIDA, M. L. O. Viscosidade de uma pasta celulósica e a resistência do papel formado. $\mathbf{O}$ Papel, v.47, n.8, p. 39-42, 1986.

DENCE, C. W.; REEVE, D. W. Pulp bleaching: principles and practice. Atlanta: Tappi Press, 1996. 800p.

DUARTE, A. P.; LACHENAL, D. Hydrogen peroxide production during oxygen bleaching of Eucalyptus globulus kraft pulp - origin of cellulose degradation. Paperi ja Puu, v.84, n.4, p. 275-277, 2002.

EIRAS, K. M. M.; COLODETTE, J. L.; CARVALHO, A. M. M. L. Estudo das causas de reversão de alvura de polpas kraft de eucalipto. Cerne, v.11, n.4, p.354-368, 2005.

EL-SHARKAWY, K.; KOSKENHELY, K.; PAULAPURO, H. The fractionation and refining of eucalyptus kraft pulps. Nordic Pulp and Paper Research Journal, v.2, n.23, p.172-180. 2008. 
FERREIRA, G. W. et al. Qualidade da celulose kraft-antraquinona de Eucalyptus dunnii plantado em cinco espaçamentos em relação ao Eucalyptus grandis e Eucalyptus saligna. Ciência Florestal, v.7, n.1, p.41-63, 1997.

GIGAC, J.; FIŠEROVÁ, M. Influence of pulp refining on tissue paper properties. TAPPI Journal, v.7, n.8, p.27-32, 2008.

GOODING, R. W.; OLSON, J. A.

Fractionation in a Bauer-McNett classifier. Journal of Pulp and Paper Science, v.27, n.12, p. 423-428, 2001.

GULLICHSEN, J.; SODERHJELM, L. On the color reversion of bleached kraft pulp. Paperi ja Puu, v.1, p.34-37, 1984.

HAKANEN, A.; HARTLER, N. Fiber deformations and strength potential of kraft pulp. Paper and Timber, n. 77, v. 5, p. 339, 1995.

KANGAS, H.; KLEEN, M. Surface chemical and morphological properties of mechanical pulp fines. Nordic Pulp and Paper Research Journal, v.19, n.2, p.191-199. 2004.

KARIM, Md. R.; ISLAM, Md. N.; MALINEN, R. O. Response of Eucalyptus camaldulensis and Acacia mangium kraft pulp in different ECF bleaching options. Wood Science and Technology, v.45, n.3, p.473-485, 2011 .

KEREKES, R. Characterizing refining action in PFI mills. TAPPI Jounal, v.4, n.3, p.9-14, 2005.

KLEEN, M.; KANGAS, H.; LAINE, C. Chemical characterization of mechanical pulp fines and fiber surface layers. Nordic Pulp and Paper Research Journal, v. 18, n.4, p.361-369, 2003.

KROGERUS, B.; FAGERHOLM, K. Fines from different pulps compared by image analysis. Nordic Pulp and Paper Research Journal, v.17, n.4, p.440-444, 2002.
LEE, H. et al. Effect of different types of fines on the properties of recycled chemical pulp.

Journal of Industrial and Engineering Chemistry, v.17, n.1, p.100-104, 2011.

LUNDIN, T. et al. Análise energética em refinação de baixa consistência de madeira de coníferas. O Papel, v.70, n.10, p.41-60, 2009.

MAIA, E. P.; COLODETTE, J. L. Efeito do conteúdo e da natureza da lignina residual na eficiência e na seletividade do branqueamento com ozônio.

Revista Árvore, v.27, n.2, p.217-232, 2003.

MILES, K. B.; KARNIS, A. Characteristics and energy consumption in refiner pulps. Journal of Pulp and Paper Science, v.21, n.11, p.j383-j399, 1995.

NAZHAD, M. M. et al. The influence of formation on tensile strength of paper made from mechanical pulps. Tappi Journal, v.83, n.12, p.63-68, 2000.

OLIVEIRA, R. L. et al. The effect of wood supply and bleaching process on pulp brightness stability. Revista Árvore, v.30, n.3, p.439-450, 2006.

PAGE, D. H. Note on the mechanism of tearing strength. Tappi Journal, v.77, n.3, p.201, 1994.

SANTOS, S.; SANSÍGOLO, C. A. Influência da densidade básica da madeira de clones de Eucalyptus grandis x Eucalyptus urophylla na qualidade da polpa branqueada. Ciência Florestal, v.7, n.1, p.53-63, 2007.

SILVA, D. J. et al. Impacto da qualidade da madeira na deslignificação, no branqueamento e nas propriedades físico-mecânicas da polpa kraft de eucalipto. O Papel, v.58, n.2, p.33-43, 1997.

TAIPALE, T. et al. Effect of microfibrillated cellulose and fines on the drainage of kraft pulp suspension and paper strength. Cellulose, v.1, n.17, p.1005-1020, 2010.

ZERONIAN, S. H.; INGLESBY, M. K. Bleaching of cellulose by hydrogen peroxide. Cellulose, v.2, p.265-272, 1995.

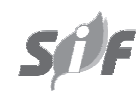

Revista Árvore, Viçosa-MG, v.37, n.1, p.181-189, 2013 